promoted as being less allergenic than animal insulins, nevertheless carry a risk of allergic reactions. To my knowledge, this is the first reported case of a generalised allergic reaction to human insulin in which reactions to highly purified monocomponent bovine and porcine insulins did not also occur.

I thank Dr S Haider for permission to report this case, Dr J S Kristenson (Novo Research Institute, Bagsvaerd, Denmark) for the determination of insulin specific IgE and IgG, Novo Laboratories Ltd for providing the allergy testing kit, and Mrs V Jones for typing the manuscript.

1 Lieberman P, Patterson R, Metz R, Lucena G. Allergic reactions to insulin. JAMA 1971;215: 1106-12.

2 Hanauer L, Batson JM. Anaphylactic shock following insulin injection. Case report and review of the literature. Diabetes $1961 ; 10: 105-9$.

3 Wiles PG, Guy R, Watkins SM, Reeves WG. Allergy to purified bovine, porcine, and human insulins. Br Med f 1983;287:531.

4 de Shazo RD, Boehm TM, Kumar D, Galloway JA, Dvorak HF. Dermal hypersensitivity reactions to insulin: correlations of three patterns to their histopathology. $\mathcal{f}$ Allergy Clin Immunol 1982;69:229-37.

5 Kumar D. Anti-insulin IgE in diabetics. 7 Clin Endocrinol Metab 1977;45:1159-64.

(Accepted 29 fanuary 1986)

Gravesend and North Kent District General Hospital, Gravesend, Kent DA11 0DG

P SILVERSTONE, MB, BS, senior house officer

Correspondence to: Medical Unit, East Surrey Hospital, Redhill, Surrey.

\section{Is there a link between iritis and diabetic autonomic neuropathy?}

The strong association reported between iritis and autonomic neuropathy in insulin dependent diabetics has been used to support the hypothesis that damage to the autonomic nervous system is immunologically mediated. ' $T$ The high prevalence of iritis found in diabetics in that study ran counter to our own clinical experience, and we therefore investigated the prevalence of the condition in patients attending the diabetic department of this hospital.

\section{Patients, methods, and results}

Tests of autonomic function ${ }^{2}$ had been performed on 385 diabetics over three years. All the diabetics, except 17 known to have died, were sent a questionnaire to elicit the relative prevalence of iritis in the groups with and without autonomic neuropathy. The questionnaire asked about painful eye conditions requiring medical consultation. We followed up a positive response by contacting the ophthalmologist or general practitioner who had seen the patient and by reviewing the medical records. We also examined the ophthalmological and diabetic records of patients who had died to ascertain whether they had had iritis.

Numbers of diabetics with iritis

\begin{tabular}{lcc}
\hline & \multicolumn{2}{c}{ Autonomic function } \\
\cline { 2 - 3 } & Abnormal & Normal \\
\hline $\begin{array}{l}\text { Diabetics who replied to questionnaire } \\
\text { Definite iritis }\end{array}$ & $3^{*}$ & 153 \\
$\begin{array}{l}\text { Undiagnosed eye condition } \\
\begin{array}{l}\text { Diabetics who had died } \\
\text { Definite iritis }\end{array}\end{array} 2^{2}$ & 163 \\
\hline $\begin{array}{c}\text { All diabetics } \\
\text { All possible cases of iritis }\end{array}$ & $1 t^{16}$ & 1 \\
\hline
\end{tabular}

^One case occurred after removal of a cataract.

fOccurred after exposure to ultraviolet light.

The questionnaire was validated by sending it to non-diabetic patients who had attended the ophthalmology department from 1980 to 1983 with a single uncomplicated episode of iritis. Of the 14 who replied, 13 responded positively to the question about a painful eye condition.

Of the 316 diabetic patients who returned the questionnaire (response rate $86 \%$ ), 33 reported a painful eye condition. Only four of these had had iritis; one had developed the condition immediately after removal of a cataract. In 27 cases we obtained a definite diagnosis of a condition other than iritis, and in two we were unable to make a diagnosis. The records of patients who had died showed one case of iritis, which had probably been caused by exposure to ultraviolet light during welding. The table summarises the results. Even when the two patients without definite diagnoses and the two patients with recognised causes of their iritis were included the prevalence of iritis in the group with autonomic neuropathy was less than $4 \%$. This was not significantly higher than that in the group with normal results of tests of autonomic function $(p=0 \cdot 13$, Fisher's exact test).

In the original report 47 diabetics aged 40 or less, all insulin dependent, had autonomic neuropathy as defined by a heart rate variability less than $10 ; 14$ gave a history of iritis. ${ }^{1}$ We identified 28 patients in our sample who fulfilled exactly these criteria; only one gave a history of iritis.

\section{Comment}

Iritis was strikingly less common in our patients with autonomic neuropathy than in those described by Guy et al. 'This was true even when the analysis was confined to patients who met the same criteria of age, insulin dependence, and abnormality of heart rate variation. We did not find iritis to be significantly more prevalent in patients with autonomic neuropathy than in patients with normal autonomic function, but the uncertainty surrounding the estimate of the odds ratio, calculated using cases of definite iritis without recognised cause (odds ratio $=2,95 \%$ confidence limits $0 \cdot 2$, 22 ), means that our data do not exclude an association between the two conditions.

Unusual patients tend to be referred to specialised clinics. ${ }^{3}$ Perhaps the reputation of Guy et al for research into diabetic neuropathy led them to study an atypical group of patients. Our failure to replicate their finding, in a large sample of patients, of a high prevalence of iritis in diabetics with autonomic neuropathy must reduce the strength of their evidence for an immune pathogenesis for diabetic autonomic neuropathy.

We are grateful to Drs L Duncan, B F Clarke, and A Adams for allowing us to study their patients and to Kate Henderson and Carol O'Neill for help in collecting the data.

1 Guy RJC, Richards F, Edmonds ME, Watkins PJ. Diabetic autonomic neuropathy and iritis: an association suggesting an immunological cause. $\mathrm{Br}$ Med $\mathcal{f}$ 1984;289:343-5.

2 Ewing DJ, Clarke BF. Diagnosis and management of diabetic autonomic neuropathy. Br Med $\mathcal{f}$ 1982;285:916-8.

3 Sackett DL. Bias in analytic research. $\mathcal{F}$ Chronic Dis 1979;32:51-63.

(Accepted 14 January 1986)

University Department of Medicine and Diabetic Department, Royal Infirmary, Edinburgh EH3 9YW

C N MARTYN, DPHIL, MRCP, lecturer

R J YOUNG, BSC, MRCP, senior registrar

D J EWING, MD, FRCP, Wellcome Trust senior lecturer

Correspondence to: Dr C N Martyn, MRC Environmental Epidemiology Unit, Southampton General Hospital, Southampton SO9 4XY.

\section{Effects of indomethacin and sulindac on blood pressure of hypertensive patients}

Some studies, ${ }^{12}$ but not others, ${ }^{3}$ have shown a pressor effect of indomethacin in patients treated for hypertension. Sulindac selectively inhibits extrarenal synthesis of prostaglandin and may not, therefore, antagonise the action of antihypertensive drugs. ${ }^{45}$ In an observer blind study we compared the effects of indomethacin and sulindac on blood pressure and symptoms in hypertensive patients who needed treatment with a non-steroidal antiinflammatory drug.

\section{Patients, methods, and results}

Twenty one patients with hypertension and coexisting joint disorders requiring treatment with a non-steroidal anti-inflammatory drug consented to the study, which was approved by the hospital ethics committee. They comprised 12 women and nine men (mean age 62 (range $40-76$ ), mean weight $80(56-108) \mathrm{kg}$ ). The antihypertensive drugs taken were kept constant throughout the study. Mean blood pressure on entry to the study was $147 / 95 \mathrm{~mm} \mathrm{Hg}$ lying and $135 / 98 \mathrm{~mm} \mathrm{Hg}$ standing. The open crossover study, with three phases, compared indomethacin ( $50 \mathrm{mg}$ twice daily), sulindac ( $200 \mathrm{mg}$ twice daily), and paracetamol ( $1 \mathrm{~g}$ four times daily); the order of treatments was varied using Williams squares. Phases of treatment lasted six weeks unless they had to be shortened because of side effects or unsatisfactory relief of symptoms. There were no washout intervals. Paracetamol was used instead of placebo because all patients required treatment for symptoms. Blood pressure, body weight, scores for pain and stiffness $(10 \mathrm{~cm}$ 
Mean (and range) results for patients who completed two weeks of treatment with all three drugs

\begin{tabular}{|c|c|c|c|c|c|}
\hline & Paracetamol & Sulindac & Indomethacin & $\mathrm{n}$ & p Value* \\
\hline \multicolumn{6}{|c|}{ Mean arterial pressure $(\mathrm{mm} \mathrm{Hg})$ : } \\
\hline Lying & $103 \cdot 8(81 \cdot 0-120 \cdot 0)$ & $109 \cdot 9(87 \cdot 0-138 \cdot 3)$ & $117 \cdot 8(102 \cdot 6-152 \cdot 0)$ & 18 & $<0.001$ \\
\hline Standing & $102 \cdot 4(78 \cdot 0-123 \cdot 6)$ & $106 \cdot 8(91 \cdot 0-138 \cdot 6)$ & $116 \cdot 4(99 \cdot 3-133 \cdot 3)$ & 18 & $<0.001$ \\
\hline Body weight $(\mathrm{kg})$ & $81 \cdot 4(56 \cdot 2-108 \cdot 8)$ & $81.5(56.4-109.5)$ & $81 \cdot 7(56 \cdot 8 \cdot 108 \cdot 5)$ & 17 & NS \\
\hline Pain score $(\mathrm{mm})$ & $63(23-98)$ & $49(20-95)$ & $46(8-82)$ & 15 & $<0.05$ \\
\hline Stiffness score $(\mathrm{mm})$ & $67(6-98)$ & $52(14-97)$ & $48(7-98)$ & 15 & $<0.05$ \\
\hline \multicolumn{6}{|c|}{ Reasons for stopping treatment: } \\
\hline Poor symptom relief & 19 & 3 & 3 & 21 & $<0.001$ \\
\hline Side effects & & 3 & 8 & 21 & $<0.01$ \\
\hline Total & 19 & 6 & 11 & 21 & $<0.001$ \\
\hline
\end{tabular}

*Significance of difference among all three treatments by analysis of variance or $\chi^{2}$ test with Yates's correction.

visual analogue scales), and counts of unused tablets were recorded at intervals of two weeks throughout the study. Blood pressure (phase $\mathrm{V}$ diastolic, right arm) was measured with a random zero sphygmomanometer by an observer who was unaware of the treatment taken. Mean arterial pressure was calculated as diastolic pressure plus one third of pulse pressure.

Many phases of treatment had to be shortened to two weeks (see table), and we therefore analysed the results after two weeks. The three drugs were compared using Friedman's two way analysis of variance followed by Wilcoxon's test for matched pairs. All data, including those for four and six weeks of treatment, were also examined by analysis of variance using multiple linear regression.

The table shows the results after two weeks. The mean arterial pressure was significantly higher with indomethacin than paracetamol $(p<0.001$ lying and standing), higher with sulindac than paracetamol (NS), and significantly highe with indomethacin than sulindac $(p<0.01$ lying and $p<0.001$ standing). Nine patients completed six weeks' treatment with both sulindac and indomethacin. Their mean arterial pressure remained significantly higher with indomethacin than sulindac, with differences after six weeks of 12.5 (SE 5.3) $\mathrm{mm} \mathrm{Hg}$ lying $(\mathrm{p}<0.05)$ and $14.6(5 \cdot 2) \mathrm{mm} \mathrm{Hg}$ standing $(\mathrm{p}<0.02)$

Analysis of the total (six week) data by multiple linear regression showed a significant $(p<0.05)$ increase in body weight during treatment with indomethacin compared with paracetamol $(0.48 \mathrm{~kg})$ and sulindac $(0.35 \mathrm{~kg})$. There was a highly significant positive correlation between changes in body weight and changes in mean arterial pressure $(p<0 \cdot 005)$. Scores for pain and stiffness during treatment with indomethacin and sulindac were similar and significantly lower than those for paracetamol.

\section{Comment}

These results show that indomethacin has a substantial and sustained pressor effect in treated hypertensive patients. The small but significant increase in body weight during treatment with indomethacin, and the positive correlation between changes in body weight and blood pressure, suggest that the pressor effect may be partly due to retention of salt and water. Sulindac did not increase blood pressure significantly compared with paracetamol. This does not exclude a pressor action of sulindac, as the $95 \%$ confidence limits indicate that it may increase lying mean arterial pressure by as much as $11.9 \mathrm{~mm} \mathrm{Hg}$, though its effect is significantly less than that of indomethacin. The data on symptoms and side effects must be viewed with caution because this was an open study, but they suggest that sulindac was as effective and well tolerated as indomethacin.

We suggest that sulindac should be preferred to indomethacin in hypertensive patients requiring a non-steroidal anti-inflammatory drug.

1 Durao U, Rico GT. Modification by indomethacin of the blood pressure lowering effect of pindolo and propranolol in conscious rabbits. Eur $\mathcal{f}$ Pharmacol 1977;43:377-81.

2 Watkins J, Abbot EC, Hensby C, Webster J, Dollery CT. Attenuation of hypotensive effect of propranolol and thiazide diuretics by indomethacin. BrMed f 1980;281:702-5.

3 Koopmans PP, Thien T, Gribnau FW. Influence of non-steroidal anti-inflammatory drugs on diuretic treatment of mild to moderate essential hypertension. Br Med f 1984;289:1492-4.

4 Salvetti A, Pedrinelli R, Alberici P, Magagna A, Abdel-Haq B. The influence of indomethacin and sulindac on some pharmacological actions of atenolol in hypertensive patients. Br $\mathcal{J}$ Clin
Phelling sulindac on some pharmacolo
Pharmacol 1984;17:108-115.

5 Steiness E, Waldorff S. Different interactions of indomethacin and sulindac with thiazides in hypertension. BrMed f 1982;285:1702-3.

(Accepted 15 fanuary 1986)

University Department of Therapeutics, Royal Hallamshire Hospital, Sheffield S10 2JF

R V LEWIS, BSC, MRCP, research fellow

J M TONER, BSC, MRCP, research fellow

P R JACKSON, MA, MRCP, lecturer

L E RAMSAY, FRCP, consultant physician and reader in clinical pharmacology and therapeutics

Correspondence to: Dr Ramsay.

\section{Antithrombin III concentration, thrombosis, and treatment with luteinising hormone releasing hormone agonist in prostatic carcinoma}

Treatment of advanced carcinoma of the prostate is based mainly on the assumption that the tumour is androgen dependent. In the past oestrogens have been used most commonly as endocrine treatment. Serious exacerbation of thromboembolic complications during oestrogen treatment, however, prompted investigation of alternative means of androgen suppression. ${ }^{1}$ In 1980 Tolis et al showed that testosterone could be suppressed by giving agonists of luteinising hormone releasing hormone, which might thus be used instead of oestrogens. ${ }^{2}$ The mechanism of the thrombogenic effect of oestrogens is unclear, but recent studies suggested that of all the effects of blood clotting induced by oestrogen treatment of prostatic cancer, the fall in plasma antithrombin III concentration is the most important. ${ }^{34}$

Before agonists of luteinising hormone releasing hormone are introduced as endocrine treatment for prostatic cancer it is essential to establish whether they influence clotting in the same way as oestrogens. We therefore measured antithrombin III concentrations in patients with carcinoma of the prostate before and during treatment with the luteinising hormone releasing hormone agonist ICI 118.630 depot to estimate the risk of thromboembolic events.

\section{Patients, methods, and results}

Thirty men aged 55-84 (mean 72) with cytologically confirmed carcinoma of the prostate were included in the study. All had locally advanced disease or distant metastases, or both. None had previously received endocrine treatment. An agonist of luteinising hormone releasing hormone (ICI $118.630 \mathrm{depot} ; 3.6 \mathrm{mg}$ ) was injected into the skin of the anterior abdominal wall at intervals of 28 days. The plasma concentration of antithrombin III was measured by a technique that uses the chromogenic substrate S-2238 (Kabi Diagnostica, Mölndal, Sweden); the normal range for adults is $80-120 \%$. Samples were taken one day before and one, two, and three months after the start of treatment. The endocrine effect of the treatment was assessed by repeated determination of plasma testosterone, luteinising hormone, and follicle stimulating hormone concentrations. The significance of mean differences in values during treatment compared with the baseline values was determined by Fisher's test.

The mean baseline concentrations of antithrombin III were within the normal range at $103 \cdot 23$ (SD 14.18)\%. No significant change took place during treatment, the concentrations one, two, and three months after the start of treatment being $100.97(14.58) \%, 103.07(13.45) \%$, and $103.20(13.92) \%$ respectively. The mean plasma concentrations of luteinising hormone and follicle stimulating hormone fell significantly $(p<0.001)$, and the mean plasma testosterone concentrations were significantly suppressed $(p<0.001)$ to values seen after castration.

\section{Comment}

Epidemiological studies and clinical experience have shown that treatment of prostatic cancer with high doses of oestrogen increases the morbidity and mortality from cardiovascular disease. Studies seeking to establish the mechanism of this phenomenon have focused on coagulation and the fibrinolytic system. Recent studies have shown that antithrombin III concentrations are reduced during oestrogen treatment in patients with carcinoma of the prostate, and this might contribute to these patients' increased incidence of cardiovascular complications. One quick way of obtaining information about the risk of undesirable effects of a new form of endocrine treatment is to study laboratory variables indicating or contribut- 